\title{
CONSERVAÇÃO PÓS-COLHEITA DE MAMÃO 'SUNRISE SOLO' COM USO DE QUITOSANA'
}

\author{
JOYCE DE QUEIRÓZ BARBOSA GALO², MARIA LUZENIRA DE SOUZA², \\ JORGE FERREIRA KUSDRA ${ }^{4}$, CLAUDIA FABRINO MACHADO MATTIUZ
}

RESUMO - O mamão é um fruto de grande importância econômica, social e nutricional. No entanto, apresenta conservação limitada devido à deterioração pós-colheita e ao rápido amadurecimento. Portanto, o objetivo deste trabalho foi utilizar soluções de quitosana associadas ao glicerol como recobrimento de mamão papaia da cultivar 'Sunrise Solo' para manter a qualidade pós-colheita e prolongar sua vida útil. Os frutos foram lavados em água potável, higienizados em solução de hipoclorito de sódio a $200 \mathrm{mg} \mathrm{L}^{-1}$ por 10 minutos e secos à temperatura ambiente. Os tratamentos consistiram na imersão ou não (controle) dos frutos em cinco concentrações $(0,25 \% ; 0,50 \% ; 0,75 \% ; 1,0 \%$ e $1,25 \%)$ de quitosana. Decorrida a secagem natural do revestimento, os frutos foram armazenados a $28 \pm 3{ }^{\circ} \mathrm{C}$ e $65-70 \%$ UR. Foi utilizado um delineamento inteiramente casualisado, utilizando-se de seis concentrações de quitosana com oito repetições, totalizando 48 frutos. Ao atingir o ponto ótimo para o consumo, avaliaram-se o teor de ácido ascórbico, de sólidos solúveis, da acidez titulável, da firmeza, a perda de massa fresca, o desenvolvimento fúngico e a vida útil. A aplicação de quitosana em mamão 'Sunrise Solo' manteve a firmeza e os teores de sólidos solúveis, de acidez titulável e de ácido ascórbico dos frutos até o ponto considerado ótimo para o consumo, em termos de aparência. A concentração de $1 \%$ de quitosana manteve a qualidade e aumentou a vida útil dos frutos em quatro dias. A quitosana inibiu o crescimento de fungos dos gêneros Cladosporium, Aspergillus e Penicillium.

Termos de Indexação: Carica papaya, biofilme, quitosana, revestimento de frutos.

\section{POSTHARVEST STORAGE OF PAPAYA 'SUNRISE SOLO' WITH THE USE OF CHITOSAN}

\begin{abstract}
Papaya is a fruit of great economic, social and nutritional importance. However, due to limited conservation presents post-harvest deterioration and rapid maturation. Therefore, the objective of this study was to use solutions of chitosan associated with glycerol as papaya coating to maintain postharvest quality and prolong its life. The fruits were washed in clean water, cleaned in sodium hypochlorite solution $200 \mathrm{mg} \mathrm{L}^{-1}$ for 10 minutes and dried at room temperature. The treatments consisted of immersion or not (control) of the fruits at five different concentrations $(0.25 \%, 0.50 \%, 0.75 \%, 1.0 \%$ and $1.25 \%)$ of chitosan. Elapsed the natural drying of the coating, the fruits were stored at $28 \pm 3{ }^{\circ} \mathrm{C}$ and $65-70 \% \mathrm{RH}$. We used a completely randomized design considering the treatments the six concentrations of chitosan with eight replications, totaling 48 fruits. Upon reaching the optimum stage for consumption, it was evaluated the content of ascorbic acid, soluble solids, titratable acidity, firmness, weight loss, fungal development and lifespan. The application of chitosan in papaya 'Sunrise Solo' maintained the firmness, the soluble solids, the titratable acidity, the ascorbic acid of the fruit to the point considered of optimal consumption, in terms of appearance. The concentration of $1 \%$ of chitosan maintained quality and increased service life of the fruit in four days. Chitosan inhibited the growth of fungi of the genera Cladosporium, Aspergillus and Penicillium. Index terms: Carica papaya, biofilm, chitosan, coating fruits.
\end{abstract}

\footnotetext{
${ }_{1}^{1}$ (Trabalho 194/13). Recebido em: 15-05-2013. Aceito para publicação em: 14-02-2014.

${ }^{2} \mathrm{Eng}^{\mathrm{a}}$. Agr ${ }^{\mathrm{a}}$., Mestre pelo Programa de Pós-graduação em Agronomia, Centro de Ciências Biológicas e da Natureza da Universidade Federal do Acre/UFAC. E-mail: joyceajc@yahoo.com.br

${ }^{3}$ Eng $^{\mathrm{a}}$. Agr ${ }^{\mathrm{a}}$., Profa. Dra. do Programa de Pós-graduação em Agronomia, Centro de Ciências Biológicas e da Natureza da UFAC. E-mail:mluzen@hotmail.com

${ }^{4}$ Eng. Agr., Prof. Dr. do Programa de Pós-graduação em Agronomia, Centro de Ciências Biológicas e da Natureza da UFAC. E-mail: kusdra@globo.com

${ }^{5}$ Profa. Dra. do Centro Universitário Moura Lacerda. Av. Dr. Oscar de Moura Lacerda, 1520. CEP: 14076-510. Ribeirão Preto-SP. E-mail: cmattiuz@gmail.com
} 


\section{INTRODUÇ̃̃O}

O mamão (Carica papaya L.) é originário da América Tropical e pertence à família Caricaceae. Segundo a FAO (2012), o Brasil ocupa a segunda posição mundial em volume de produção $(17 \%) \mathrm{e}$ a terceira em área colhida (36.585 ha), com uma produção, em 2011, de 1,9 milhão de toneladas.

A cultura do mamoeiro tem expressiva participação na produção nacional da fruticultura. É a terceira fruta mais consumida no País e apresenta grande relevância econômica e social, principalmente na geração de emprego e renda. Segundo dados do AGRIANUAL (2011), os principais estados produtores em 2008 foram: Bahia (902 mil toneladas), Espírito Santo (630 mil toneladas), Rio Grande do Norte (106 mil toneladas) e Ceará (100 mil toneladas).

No Estado do Acre, as condições edafoclimáticas são favoráveis para a produção de mamão. Porém, este apresenta baixa produtividade $\left(12,9 \mathrm{t} \mathrm{ha}^{-1}\right)$, e a quantidade produzida em 2008 (3.054 t) foi insuficiente para atender à demanda local (AGRIANUAL, 2011).

De modo geral, são perdidos $30 \%$ do total produzido de frutas devido às alterações físicas e químicas após a colheita, como modificações da textura, aroma, sabor e cor. Além disso, por ser fruto climatério, apresenta aumento da taxa respiratória, produção autocatalítica de etileno e alterações substanciais no amadurecimento tais como: cor, sabor, amaciamento e produção de compostos voláteis aromáticos (PEREIRA et al., 2006).

Dentre os procedimentos aplicados em frutas tropicais para prolongar a vida útil pós-colheita, o uso de compostos naturais ou biodegradáveis, não tóxicos, derivados de animais ou plantas, que apresentem efeito fungistático ou induzam a resistência natural das plantas, tem-se destacado nos trabalhos de conservação de produtos vegetais (BAUTISTA-BAÑOS et al., 2006), sendo a quitosana um desses produtos.

A quitosana é um polissacarídeo obtido da desacetilização da quitina, presente principalmente em insetos e invertebrados marinhos, com ação fungistática sem, porém, causar reações de toxicidade ao organismo humano. Associada ao glicerol, um composto orgânico de propriedades solventes e umectantes, adere-se melhor aos frutos, formando um filme natural e permeável às trocas gasosas realizadas pelo vegetal, permitindo a redução do metabolismo do produto e o prolongamento de sua vida útil (TERRY; JOYCE, 2004; CAMILI et al., 2007; LARANJEIRA; FÁVERE, 2009).
O objetivo deste trabalho foi utilizar solução de quitosana associada ao glicerol para o recobrimento do mamão papaia a fim de manter a qualidade póscolheita e prolongar sua vida útil.

\section{MATERIAL E MÉTODOS}

Frutos de mamoeiro da cultivar 'Sunrise Solo' foram obtidos no período de julho a agosto de 2011, em pomar comercial da propriedade Boa Vista, localizada no município de Porto Acre-AC. Estes foram colhidos no estádio 2 de maturação, com $25 \%$ de coloração amarela, deixando-se cerca de $3 \mathrm{~cm}$ do pedúnculo, para reduzir a incidência de doenças. Em seguida, foram transportados em caixas de papelão até o laboratório da Unidade de Tecnologia de Alimentos (UTAL), da Universidade Federal do Acre (UFAC), em Rio Branco - AC, onde se efetuou a seleção para obtenção de lotes uniformes, eliminando-se os frutos com defeitos, dano mecânico e sintomas de doenças. Posteriormente, foram lavados em água potável, imersos em solução de hipoclorito de sódio a $200 \mathrm{mg} \mathrm{L}^{-1}$ por 10 minutos e secos à temperatura ambiente.

A quitosana comercial empregada (densidade de $0,6 \mathrm{~g} \mathrm{~mL}^{-1}$, viscosidade de 20 a $50 \mathrm{cps}$ e grau de desacetilização de $90 \%$ ) foi dissolvida em água, com auxílio de $1 \%$ de ácido láctico, sob aquecimento e agitação até completa homogeneização para obter as soluções a $0,25 \% ; 0,50 \% ; 0,75 \% ; 1,0 \%$ e $1,25 \%$ de quitosana e, em cada uma, adicionados $3 \%$ de glicerol e ajuste de $\mathrm{pH}$ a 5,6 com $\mathrm{NaOH}$ 0,1 M.

Os frutos foram imersos em diferentes concentrações de quitosana, durante 1 minuto, em seguida retirados e postos para escorrer. A cura (polimerização) do filme de quitosana deu-se espontaneamente, devido à evaporação da água (solvente). Decorrida a secagem natural da cobertura, os frutos foram dispostos em bandejas de poliestireno expandido e armazenados à temperatura de $28 \pm$ $3^{\circ} \mathrm{C}$ e $65-70 \%$ UR para análises no ponto ótimo de consumo, que foi até o $13^{\circ}$ dia.

Foi utilizado um delineamento inteiramente casualisado, considerando-se como tratamento seis concentrações de quitosana $(0 \% ; 0,25 \%$; $0,50 \% ; 0,75 \% ; 1,0 \%$ e $1,25 \%$ ) com oito repetições, totalizando 48 frutos.

Ao atingirem o ponto considerado ótimo para o consumo, em termos de aparência, os frutos foram submetidos às análises quanto aos teores de sólidos solúveis ( $\mathrm{SS}$ ), de acidez titulável em ácido cítrico (AT), de ácido ascórbico (AA), realizadas conforme a metodologia do Instituto Adolfo Lutz (2005), e à firmeza. 
A firmeza foi medida nos frutos inteiros sem casca, utilizando-se de Texturômetro Stevens - LFRA Texture Analyser (penetrômetro analógico manual), com a ponta de prova -TA 9/1000 e velocidade de penetração de $2,0 \mathrm{~mm} / \mathrm{seg}$, na profundidade de $20 \mathrm{~mm}$, tomando-se duas leituras na região equatorial, em ambos os lados de um mesmo fruto.

A vida útil foi determinada em função dos dias necessários para os frutos atingirem o $5^{\circ}$ estádio de maturação e apresentarem condições de sanidade e aparência adequadas para sua comercialização/ consumo.

A porcentagem de perda de massa fresca foi determinada pela diferença entre a massa inicial do fruto e a obtida a cada intervalo de tempo de amostragem (massa final), utilizando-se de balança digital, semianalítica, com precisão de 0,05 g. Avaliaram-se, diariamente, seis frutos (parcela) de cada tratamento até o amadurecimento. Para a análise microbiológica de fungos, um fruto de cada tratamento foi submetido à técnica de amostragem com "swabs" (SILVA et al., 2007).

Os dados coletados foram submetidos à análise de regressão, em função dos tratamentos quantitativos (concentrações de quitosana), e comparado o efeito dos tratamentos com ausência (controle) e presença $(0,25 \% ; 0,50 \% ; 0,75 \% ; 1,00 \%$ e $1,25 \%$ ) de quitosana, por contrastes ortogonais (NOGUEIRA, 2004).

\section{RESULTADOS E DISCUSSÃO}

Verifica-se, na Figura 1, que os teores de sólidos solúveis aumentaram até uma determinada concentração $(0,72 \%)$ de quitosana.

Pela análise por contrastes ortogonais (Tabela 1), confirma-se o efeito significativo e positivo nesta variável (sólidos solúveis foi maior) pela presença de quitosana, ou seja, os frutos tornaram-se mais doces.

Dang et al. (2010), ao avaliarem o efeito do revestimento de quitosana em cerejas, também verificaram incremento nos teores de sólidos solúveis dos frutos revestidos comparados com o tratamentocontrole.

Em relação à acidez titulável, verificamse maiores valores proporcionais ao aumento da concentração de quitosana aplicada (Figura 2). Pode-se inferir que os tratamentos com quitosana desaceleraram o processo normal de amadurecimento dos mamões, visto que a diminuição da acidez está associada ao consumo de ácidos no processo respiratório, em decorrência da maturação (CHITARRA; CHITARRA, 2005).

Souza et al. (2011), estudando mangas
'Tommy Atkins' tratadas com quitosana, armazenadas sob temperatura ambiente $\left(23 \pm 2{ }^{\circ} \mathrm{C}\right.$ e $65 \pm 5 \%$ UR), também verificaram aumento na acidez titulável, devido ao retardo do amadurecimento.

Para os teores de ácido ascórbico, verificouse crescimento proporcional à concentração de quitosana aplicada (Figura 3) com valor máximo obtido com aplicação de $1,09 \%$ a 1,25\% de quitosana. Segundo Chitarra e Chitarra (2005), o ácido ascórbico é um composto antioxidante sintetizado pelas frutas e hortaliças em quantidades variáveis, de acordo com a espécie, cultivar, fatores ambientais e grau de maturação, e que tende a diminuir com os processos de amadurecimento e senescência. Isto significa que os frutos tratados com quitosana retardaram o amadurecimento quando comparados com os do controle.

Dang et al. (2010) verificaram que o teor de ácido ascórbico em cerejas revestidas com quitosana foi maior do que as do controle. Segundo os autores, a redução da perda de ácido ascórbico em cerejas revestidas pode ser devida à permeabilidade do revestimento de quitosana que reduziu a atividade das enzimas e impediu a oxidação do ácido ascórbico.

Observou-se que os revestimentos de quitosana contribuíram para a manutenção da firmeza, da polpa dos frutos (Figura 4). Os maiores valores obtidos em mamões revestidos (Tabela 1) e na concentração máxima de quitosana $(1,25 \%)$ indicam tendência de resposta desta variável para maiores concentrações de quitosana.

Embora o tratamento dos frutos com quitosana a $1,25 \%$ tenha permitido obter maior firmeza, observou-se que este alterou negativamente o amadurecimento, prejudicando, desta forma, a qualidade final dos frutos.

Cerqueira et al. (2011) observaram fenômeno semelhante ao estudar quitosana em goiabas, em que o tratamento com $6 \%$ interferiu na perda normal de firmeza; provavelmente, isso se deve à excessiva restrição às trocas gasosas entre o tecido vegetal e o ambiente externo, resultando alteração no metabolismo da fruta, provocando redução dos processos de degradação da parede celular e quebra de pectinas, mantendo, desta forma, maior firmeza do tecido.

Diferenças na firmeza, em frutos tratados com quitosana, também foram observadas por BautistaBaños et al. (2003), González-Aguilar et al. (2009) e Souza et al. (2011), que obtiveram maior firmeza em frutos revestidos com quitosana.

Todas as concentrações de quitosana prolongaram a vida útil do mamão, proporcionando aumentos de $1(0,25 \%), 2$ (0,50\%), $3(0,75 \%), 4$ 
$(1,00 \%)$ e $5(1,25 \%)$ dias, respectivamente (Figura 5), em relação à testemunha. Porém, a concentração de $1 \%$ de quitosana manteve a melhor qualidade dos frutos, sem alterar nenhuma característica externa do mamão, confirmando esta como a concentração ideal. Certamente, os tratamentos com quitosana formaram uma barreira sobre a superfície dos frutos, reduzindo suas trocas gasosas e retardando seus processos metabólicos de amadurecimento.

Comparando a aplicação ou não de quitosana, independentemente de suas concentrações, verificase a maior perda de massa fresca nos tratamentos com presença de quitosana (Tabela 1). Esta situação pode ser devido ao fato de os frutos tratados com quitosana terem permanecido com a cor verde por mais tempo, pelo retardamento do amadurecimento e, nesta condição, perderem mais massa fresca, decorrente das maiores taxas de respiração e transpiração.

Estes dados vão de encontro aos obtidos por Lin et al. (2008) ao avaliarem o efeito do revestimento de quitosana na qualidade de peras 'Yali' e verificarem redução significativa da perda de massa fresca em peras revestidas com 1,5\% de quitosana em comparação com as não revestidas, durante o armazenamento a $25^{\circ} \mathrm{C}$ e $80-90 \%$ de UR.

Chien et al. (2007a) observaram menor perda de massa fresca $(10,27 \%)$ em mangas minimamente processadas, tratadas com quitosana (2\%), comparadas com o tratamento-controle $(19,86 \%)$.

Verificou-se a maior contagem de fungos no tratamento-controle (ausência de quitosana) (Tabela
1), e menores contagens nos tratamentos com maior porcentagem de quitosana (Tabela 2). As contagens de fungos foram influenciadas pela concentração de quitosana e pelo tempo de armazenamento (interação significativa), observando-se contagem mínima (21 UFC. $\mathrm{mL}^{-1}$ ) no tratamento com $1,25 \%$ de quitosana, aos 3 dias de armazenamento, e contagem máxima (577 UFC.mL $\left.\mathrm{m}^{-1}\right)$ na ausência de quitosana $(0 \%)$, aos 6 dias de armazenamento. Foram identificados os gêneros Cladosporium, Aspergillus e Penicillium nos frutos e confirmado o efeito fungistático da quitosana, embora já relatado em outros trabalhos (CHIEN et al., 2007b; HERNÁNDEZ-MUÑOZ et al., 2006; VARGAS et al., 2006) e, neste trabalho, observado na concentração máxima de quitosana (1,25\%). Pela análise de contrastes ortogonais (Tabelas 1 e 2), verificou-se que a quitosana foi eficiente em reduzir a contagem de fungos.

Gonzáles-Aguilar et al. (2009) também comprovaram a eficiência da quitosana na inibição do crescimento de fungos ao estudarem o efeito de revestimentos de quitosana em mamões minimamente processados, armazenados por 14 dias a $5{ }^{\circ} \mathrm{C}$. Segundo Bautista-Baños et al. (2006), a quitosana apresenta efeito de controlar microrganismos patogênicos e de ativar a defesa, induzindo e/ou inibindo diferentes atividades bioquímicas durante a interação planta-patógeno. Zhu et al. (2008), trabalhando com mangas cv. Tainong cobertas com quitosana a 0,$5 ; 1,0$ e 2,0\%, afirmam que a quitosana inibiu o progresso de doença nos frutos inoculados com Colletotrichum gloeosporioides.

TABELA 1 - Variáveis físicas e químicas de mamão ‘Sunrise Solo’ obtidas em resposta à ausência e presença de quitosana, no ponto ótimo de consumo, em experimento no delineamento inteiramente casualizado, avaliadas por contrastes ortogonais.

\begin{tabular}{lcc}
\hline Variáveis & \multicolumn{2}{c}{ Quitosana (\%) } \\
\cline { 2 - 3 } Sólidos solúveis ( ${ }^{\circ}$ Brix) & Ausência & Presença \\
Acidez titulável (g/100 ácido cítrico) & $11,71 \mathrm{~b}$ & $12,58 \mathrm{a}$ \\
Ácido ascórbico (mg $\left.100 \mathrm{~g}^{-1}\right)$ & $0,19 \mathrm{~b}$ & $0,20 \mathrm{a}$ \\
Firmeza (N) & $9,30 \mathrm{~b}$ & $14,28 \mathrm{a}$ \\
Perda de massa fresca (\%) & $13,18 \mathrm{~b}$ & $18,77 \mathrm{a}$ \\
Fungos (UFC. $\left.\mathrm{mL}^{-1}\right)$ & $0,84 \mathrm{~b}$ & $0,91 \mathrm{a}$ \\
\hline
\end{tabular}


TABELA 2 - Contagem de fungos em função da interação entre concentrações de quitosana e tempo de armazenamento de frutos de mamão 'Sunrise solo'

\begin{tabular}{cccccc}
\hline Concentração & \multicolumn{5}{c}{ Tempo (dias) } \\
\cline { 2 - 6 } de quitosana (\%) & 0 & 3 & 6 & 9 & 12 \\
\hline & \multicolumn{5}{c}{ Contagem de fungos/UFC.mL - $^{-1}$} \\
\hline 0,00 & 493 & 367 & 577 & 160 & 313 \\
0,25 & 483 & 243 & 520 & 117 & 250 \\
0,50 & 377 & 183 & 273 & 110 & 160 \\
0,75 & 307 & 127 & 257 & 123 & 120 \\
1,00 & 257 & 107 & 140 & 103 & 110 \\
1,25 & 193 & 21 & 133 & 48 & 35 \\
\hline
\end{tabular}

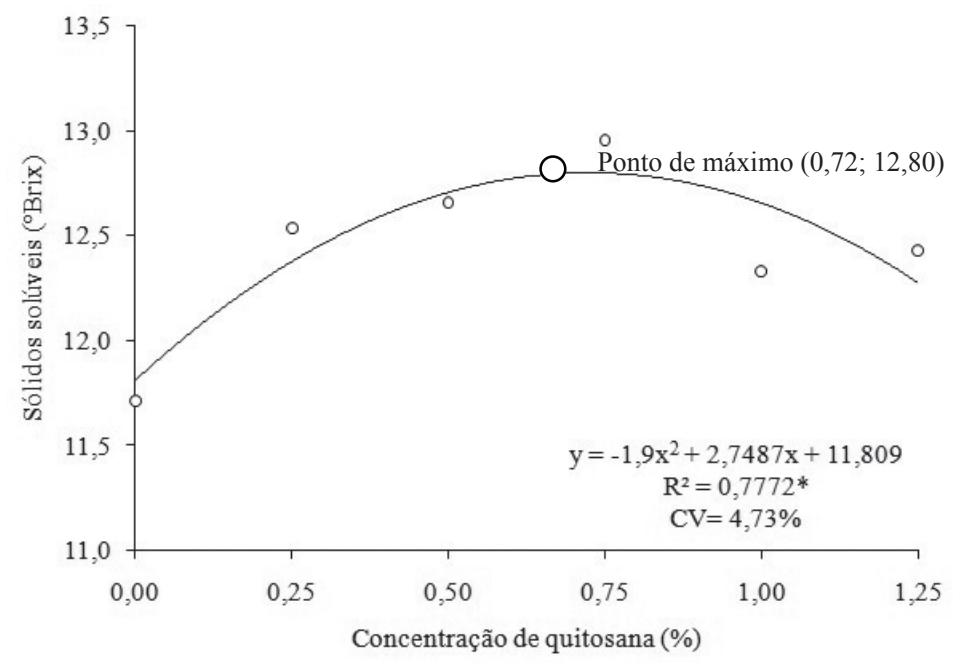

FIGURA 1 - Sólidos solúveis de mamão ‘Sunrise Solo' tratado com diferentes concentrações de quitosana e analisado no ponto ótimo ao consumo.

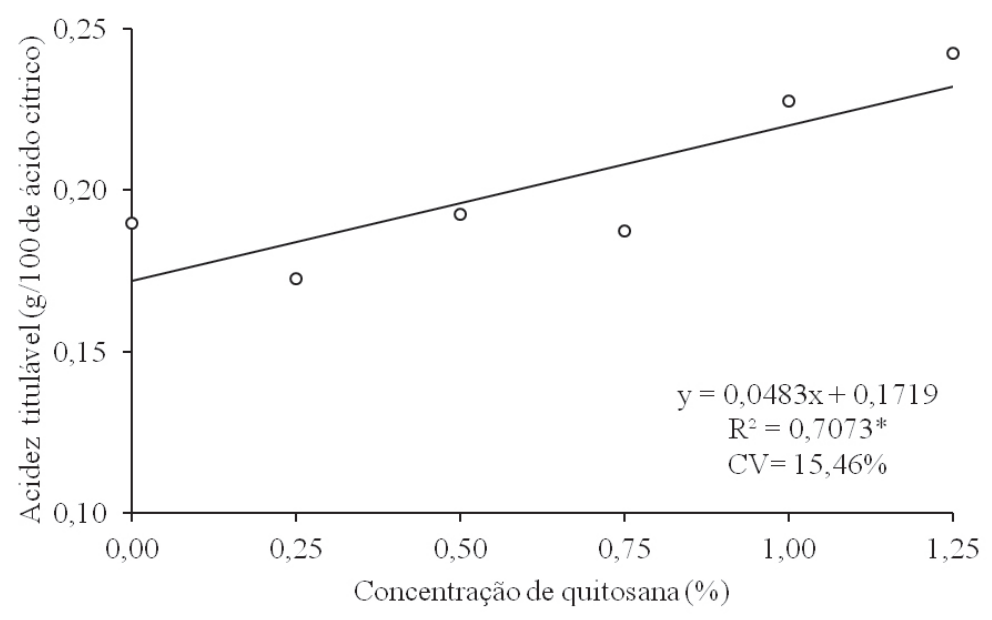

FIGURA 2 - Acidez titulável, em ácido cítrico, do mamão ‘Sunrise Solo’ tratado com diferentes concentrações de quitosana e analisado no ponto ótimo ao consumo. 


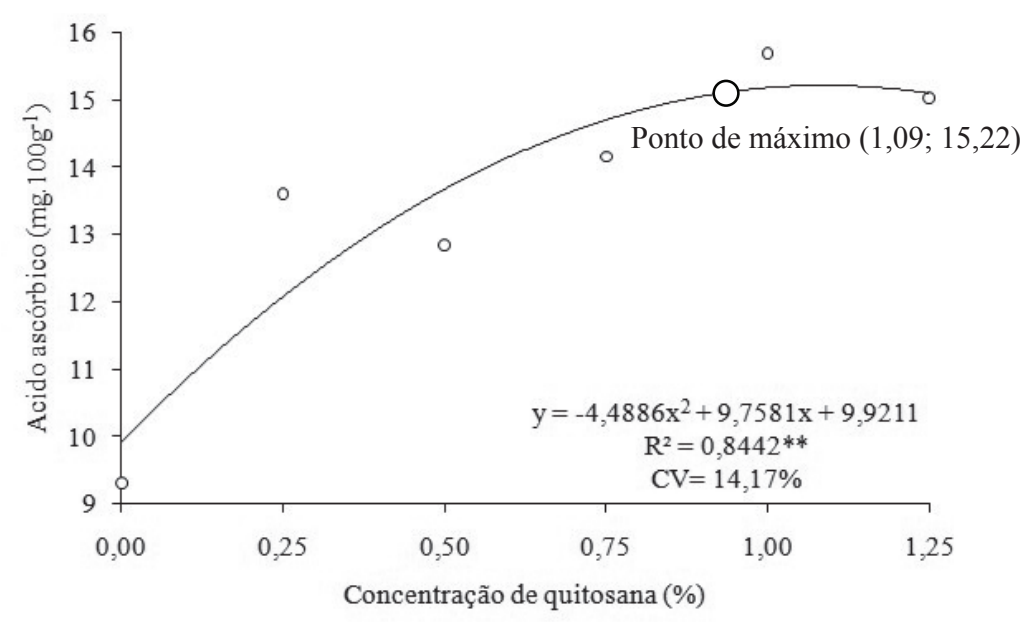

FIGURA 3 - Ácido ascórbico de mamão 'Sunrise Solo' tratado com diferentes concentrações de quitosana e analisado no ponto ótimo ao consumo.

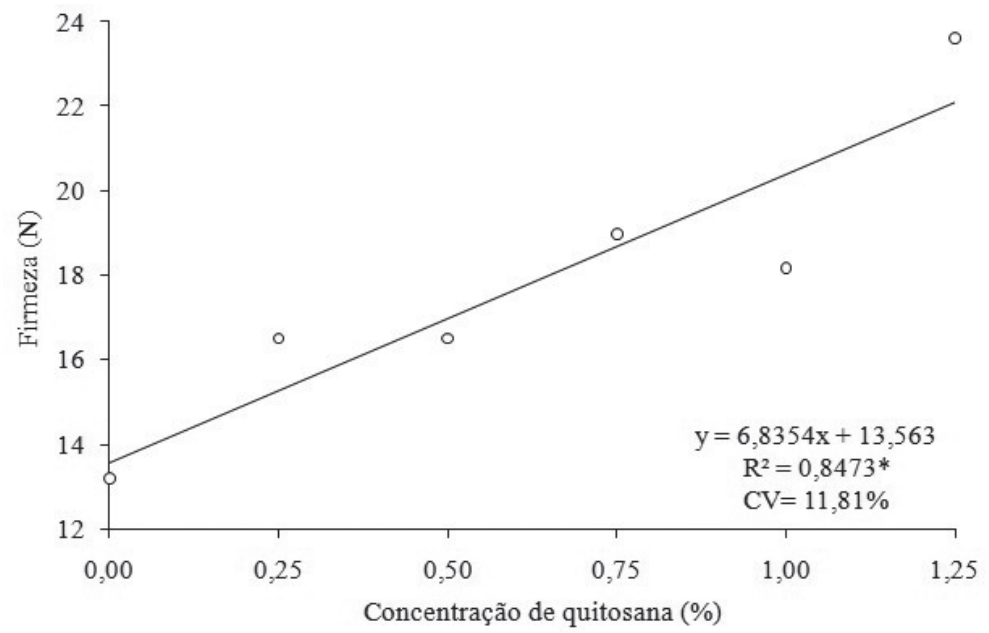

FIGURA 4 - Firmeza de mamão 'Sunrise Solo' tratados com diferentes concentrações de quitosana e analisada no ponto ótimo ao consumo.

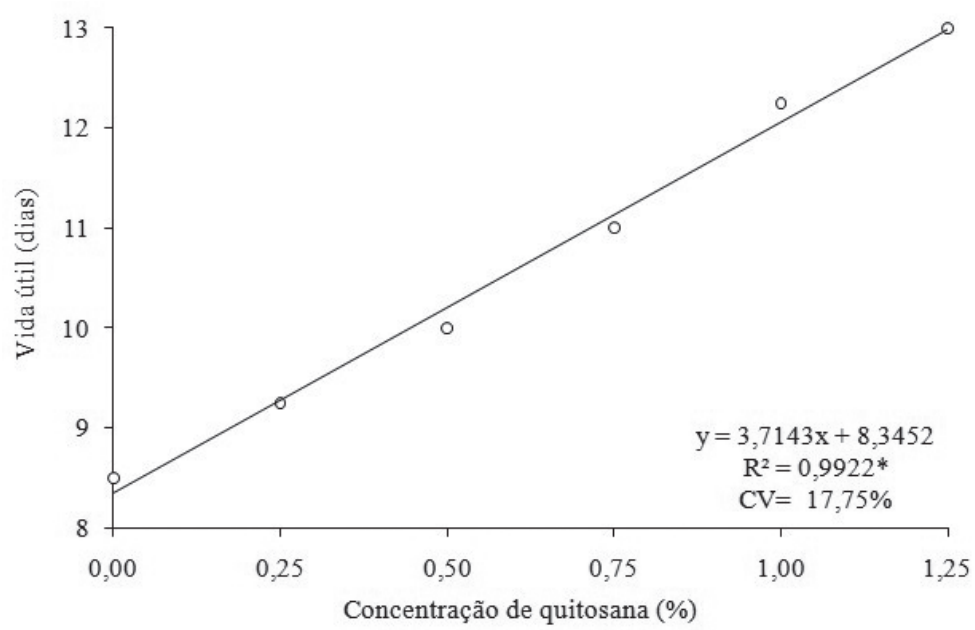

FIGURA 5 - Vida útil do mamão ‘Sunrise Solo’ tratado com diferentes concentrações de quitosana. 


\section{CONCLUSÕES}

1-A quitosana como cobertura em mamão 'Sunrise Solo' manteve a firmeza e os teores de sólidos solúveis, de acidez titulável e de ácido ascórbico dos frutos até o ponto considerado ótimo ao consumo.

2-A concentração de $1 \%$ de quitosana manteve a qualidade e aumentou a vida útil dos frutos em quatro dias.

3-A quitosana inibiu o crescimento de fungos dos gêneros Cladosporium, Aspergillus e Penicillium.

\section{REFERÊNCIAS}

AGRIANUAL: anuário da agricultura brasileira. São Paulo: FNP, 2010. 549p.

BAUTISTA-BAÑOS, S.; HERNÁNDEZ-LÓPEZ, M.; BOSQUEZ-MOLINA, E.; WILSON, C. L. Effects of chitosan and plant extracts on growth of Colletotrichum gloeosporioides, anthracnose levels and quality of papaya fruit. Crop Protection, Guildford, v. 22, p.1087-1092, 2003.

BAUTISTA-BAÑOS, S.; HERNÁNDEZLAUZARDO, A. N.; VALLE, M. G. V.; HERNÁNDEZ-LÓPEZ, M.; BARKA, E. A.; BOSQUEZ-MOLINA, E.; WILSON C. L. Chitosan as a potential natural compound to control pre and postharvest diseases of horticultural commodities. Crop Protection, London, v. 25, p.108-118, 2006.

CAMILLI, E. C.; BENATO, E. A.; PASCHOLATI, S. F.; Cia, P. Avaliação de quitosana, aplicada em póscolheita, na proteção de uva 'Itália' contra Botrytis cinerea. Summa Phytopathologica, Botucatu, v. 33, p. 3, p.215-221, 2007.

CERQUEIRA, T. S.; JACOMINO, A. P.; SASAKI, F. F.; ALLEONI, A. C. C. Recobrimento de goiabas com filmes proteicos e quitosana. Bragantia, Campinas, v. 70, n. 1, p. 216-221, 2011.

CHIEN, P.; SHEU, F.; YANG, F. Effects of edible chitosan coating on quality and shelf life of sliced mango fruit. Journal of Food Engineering, Taipei, v. 78, p. 225-229, 2007a.
CHIEN, P.; SHEU, F.; LIN, H. Coating citrus (Murcott tangor) fruit with low molecular weight chitosan increases postharvest quality and shelf life. Food Chemistry, Taipei, v. 100, p.1160-1164, 2007 b.

CHItARRA, M. I. F.; CHITARRA, A. B. Póscolheita de frutas e hortaliças: fisiologia e manuseio. 2. ed. Lavras: UFLA, 2005.

DANG, Q. F.; YAN, J. Q.; LI, Y.; CHENG, X. J.; LIU, C. S.; CHEN, X. G. Chitosan acetate as an active coating Material and Its Effects on the Storing of Prunus avium L. Journal of Food Science, Chicago, v. 75, n. 2, p. 129, 2010.

FAO - Food and Agriculture Organization of United Nations. Disponível em: <http:// www.fao.org $>$. Acesso em: 28 fev. 2012.

GONZALEZ-AGUILAR, G.; VALENZUELA-SOTO, E.; LIZARDI-MENDOZA, GOYCOOLEA, F.; MARTPINEZ-TÉLLEZ, M.; VILLEGAS-OCHOA, M.; MONROY-GARCÍA, I.; AYALA-ZAVALA, J. F. Effect of chitosan coating in preventing deterioration and preserving the quality of fresh-cut papaya 'Maradol'. Journal of Food Science Agriculture, Sonora, v. 89, p. 15-23, 2009.

HERNÁNDEZ-MUÑOZ, P.; ALMENAR, E.; OCIO, M. J.; GAVARA, R. Effect of calcium dips and chitosan coatings on postharvest life of strawberries (Fragaria x Ananassa). Postharvest Biology and Technology, Valencia, v. 39, p. 247-253, 2006.

INSTITUTO ADOLFO LUTZ. Métodos físicoquímicos para análise de alimentos. 2005. Disponível em: <http://www.ial.sp.gov.br/index. php? option $=$ com remository \& Itemid $=0 \&$ func $=$ select\&orderby $=1>$. Acesso em: 20 ago. 2012.

LARANJEIRA, M. C. M.; FÁVERE, V. T. Quitosana: biopolímero funcional com potencial industrial biomédico. Química Nova, Florianópolis, v. 32, n. 3, p. 672-678, 2009.

LIN, L.; WANG, B.; WANG, M.; CAO, J.; ZHANG, J.; WU, Y.; JIANG, W. Effects of a chitosanbased coating with ascorbic acid on post-harvest quality and core browning of 'Yali' pears (Pyrus bertschneideri Rehd.), Journal of the Science of Food and Agriculture, Beijing, v. 88, p. 877-884, 2008. 
NOGUEIRA, M. C. S. Orthogonal Contrasts: definitions and concepts, Scientia Agricola, Piracicaba, v. 61, n. 1, p. 118-124, 2004.

PEREIRA, M. E. C.; SILVA, A. S. da; BISPO, A. S. da R.; SANTOS, D. B. dos; SANTOS, S. B. dos; SANTOS, V. J. dos. Amadurecimento de mamão Formosa com revestimento comestível à base de fécula de mandioca. Ciência e Agrotecnologia, Lavras, v. 30, n. 6, p.1116-1119, 2006.

SILVA, N. da; JUNQUEIRA, V. C. A.; SILVEIRA, A. de F. N.; TANIWAKI, M. H.; SANTOS, R. F. S. dos; GOMES, R. A. R. Manual de métodos de análise microbiológica de alimentos. 3. ed. São Paulo: Livraria Varela, 2007.

SOUZA, M. L. de; MORGADO, C. M. A.; MARQUES, K. M.; MATTIUZ, C. F. M.; MATTIUZ, B. Pós-colheita de mangas 'Tommy Atkins' recobertas com quitosana. Revista Brasileira de Fruticultura, Jaboticabal, p. 337-343, 2011. Volume especial.
TERRY, L. A.; JOYCE, D. C. Suppression of grey mould on strawberry fruit with the chemical plant activator acibenzolar. Pest Management Science, Hoboken, v.56, p. 989-992, 2004.

VARGAS, M.; ALBORS, A.; CHIRALT, A.; GONZÁLEZ-MARTÍNEZ, C. Quality of cold-stored strawberries as affected by chitosan-oleic acid edible coatings. Postharvest Biology and Technology, Valencia, v. 41, p. 164-171, 2006.

ZHU, X.; WANG, Q.; CAO, J.; JIANG, W. Effects of chitosan coating on postharvest quality of mango (Mangifera indica L. Cv. Tainong) fruits. Journal of Food Processing \& Preservation, Westport, v.32, n.5, p.770-784, 2008. 